\title{
Regulation of ARL2 in colorectal cancer cell proliferation and tumorigenicity, and its negative association with AXL
}

\author{
XUNLEI PANG ${ }^{1,2^{*}}$, YANHONG WANG $^{2 *}$, BEI MIAO $^{2}$, SUJUAN FEI $^{2}$ and WEICHANG CHEN ${ }^{1}$ \\ ${ }^{1}$ Department of Gastroenterology, The First Affiliated Hospital of Soochow University, Suzhou, Jiangsu 215008; \\ ${ }^{2}$ Department of Gastroenterology, The Affiliated Hospital of Xuzhou Medical University, Xuzhou, Jiangsu 221004, P.R. China
}

Received June 2 2020; Accepted December 4, 2020

DOI: $10.3892 / 01.2021 .12457$

\begin{abstract}
Colorectal cancer (CRC) is the third most common malignant disease in adults. ADP ribosylation factor-like GTPase 2 (ARL2) is crucial for controlling the dynamics of microtubules and mitochondrial functions. However, the biological function of ARL2 in CRC remains unclear. The present study was performed to identify the expression level and functional role of ARL2 in CRC. A total of 19 CRC and 3 normal healthy colorectal tissues were collected. Furthermore, ARL2 expression was analyzed in healthy colorectal and CRC tissues by immunohistochemistry (IHC). ARL2 overexpression and knockdown was achieved using lentiviral vectors and plasmid transfection in HCT8 and HCT116 cells. The protein and mRNA expression levels of ARL2 and AXL were analyzed using western blot and reverse transcription-quantitative PCR in ARL2 knockdown and ARL2 overexpressing HCT8 and HCT116 cells. Cell Counting Kit-8, colony formation, wound healing, and Matrigel assays were used to investigate the biological functions of ARL2. Taken together, ARL2 protein expression level was upregulated in CRC tissues. Furthermore, ARL2 overexpression decreased proliferation and weakened the colony-formation abilities of the CRC cells, as well as their migratory and invasive abilities. ARL2 interference enhanced proliferation and colony-formation rates of the CRC cells, as well as their migratory and invasive abilities. ARL2 regulated $\mathrm{CRC}$ proliferation and tumorigenicity and was negatively associated with AXL. The results of the present study suggested that the proliferation, migration and tumorigenicity of the CRC cells could be inhibited by ARL 2 overexpression. The latter may be used as a predicted and potential therapeutic target for CRC.
\end{abstract}

Correspondence to: Dr Weichang Chen, Department of Gastroenterology, The First Affiliated Hospital of Soochow University, 188 Shizi Street, Suzhou, Jiangsu 215008, P.R. China

E-mail: weichangchen2020@163.com

*Contributed equally

Key words: ARL2, colon cancer, AXL, tumorgenicity

\section{Introduction}

As the third most common malignant disease in the world, colorectal cancer (CRC) exhibits a $2.27 \%$ cumulative risk of onset between 0-74 years of age (1). In China, CRC is also one of the 5 most common types of cancer, and poses a significant disease burden in the aging population (2). The 5-year relative survival rate for Chinese patients with CRC has increased from 47.2 to $56.9 \%$ in the past 10 years. However, the possible of mortality rate of CRC has remained at $50 \%$ (3). The overall prognosis of CRC was improved from the use of adjuvant chemotherapy and the advance of resection. However, even in the early stages of $\mathrm{CRC}$, recurrence following surgery remains the primary, and ultimate, cause of death (4). The development of a potential therapeutic target, that can aid the detection and treatment of CRC, is important for the prevention of this disease.

Multiple mitochondrial functions, including motility, mitochondrial morphology, asymmetric division and maintenance of ATP levels $(5,6)$ are necessary for the function of phosphodiesterase- $\delta$. The ADP ribosylation factor-like GTPase2 (ARL2) assists in the release of Ras (6). Multiple cell signaling pathways and various cellular functions are regulated by Ras, including proliferation, differentiation, vesicle transport, nuclear assembly, and the regulation of the cytoskeleton are included (7). Several studies have shown the contribution of a Ras mutation, such as KRAS-4B, in the development of human tumors (8-10). It has been shown that $\sim 21 \%$ of all human cancers, and $\sim 30,45$, and $90 \%$ of lung, colon and pancreatic cancers, respectively, originate due to activating mutations in the Ras family of enzymes (8). It has also been found that the regulation of ARL2 protein expression could alter the duration and degree of numerous types of cancer, and the cell migratory and invasive abilities of cancer cells $(11,12)$. ARL2 has been shown to regulate p53 localization, resulting in a chemoresistant phenotype in breast cancer cells (13). As a prognostic marker, ARL2 mRNA expression was significantly elevated in hepatocellular tumors (14). In addition, ARL2 acts as an oncogene in cervical cancer (15). However, the functional role of ARL2 in CRC is not clear.

AXL is a member of the TAM group of receptors, an inhibitor of the cytokine receptor-mediated macrophage/monocyte activation and promoter of apoptotic cell removal. In addition, 
AXL is an oncotarget in human CRC (16). Silencing of AXL gene expression suppresses proliferation, migration and survival in CRC cells (16). In CRC, AXL is overexpressed and its elevated protein expression promotes migration, invasion and epithelial-mesenchymal transition of CRC cell lines (17).

The expression and function of ARL2 in CRC was investigated in the current study. It was found that ARL2 overexpression or knockdown regulated the proliferation, colony formation, migration and invasion abilities of CRC cells and it had a negative association with AXL.

\section{Materials and methods}

Patients and samples. The patient samples were collected from The Affiliated Hospital of Xuzhou Medical University (Jiangsu, China) from January to June in 2019, including 19 colon samples (6 well-differentiated, 6 moderately differentiated and 7 poorly differentiated cases). All patients with CRC underwent surgical resection and histological diagnosis was verified by 2 gastroenterologists (Pathology Department of The Affiliated Hospital of Xuzhou Medical University) (Xuzhou, China). Patients with CRC who had undergone preoperative radiotherapy and chemotherapy were excluded. A total of 19 colon cancer tissues and 3 healthy colorectal tissues (from partial resection in patients with colorectal trauma. As a regional scientific research and medical institution, some patients suitable for being assigned to normal controls were informed and signed informed consent before or after surgery were used for immunohistochemistry (IHC) analysis. Surgical resection was performed in all patients with colon cancer, and the histological diagnosis was confirmed according to 2016 World Health Organization (WHO) guidelines (18). The present study was approved by the Medical Ethics Committee of the Hospital of The Affiliated Hospital of Xuzhou Medical University (Jiangsu, China), and written informed consent was provided from each patient for his/her participation in the study (approval no. XYFY2019-KL157-01). The clinical characteristics of the 19 patients with colon cancer are listed in Table I.

IHC assay. IHC analysis of ARL2 was performed on 19 colon cancer and 3 healthy colorectal tissues. The samples were fixed in $10 \%$ neutral buffered formalin for $\sim 3-24 \mathrm{~h}$ at room temperature, dehydrated in gradient alcohol solution $(30,50,70,80,90,95,100,100 \%$ alcohol each for $30 \mathrm{~min})$ and paraffin-embedded. Paraffin-embedded tissues were cut into $\sim 3-\mu$ m thick continuous sections. Routine dewaxing, antigen retrieval (microwave thermal retrieval) and quenching of endogenous peroxidase activity with $3 \% \mathrm{H}_{2} \mathrm{O}_{2}$ at room temperature was performed on the slices. After blocking with 5\% goat serum [cat. no. abs933; Absin (Shanghai) Biotechnology Co., Ltd.] at room temperature for $\sim 1 \mathrm{~h}$, primary antibody (anti-ARL2; 1:100; cat. no. 188322; Cell Signaling Technology, Inc.) was added to the sections and incubated overnight at $4^{\circ} \mathrm{C}$. The substrate [primary reactant amplifier; cat. no. abs957; Absin (Shanghai) Biotechnology Co., Ltd.] for the secondary antibody was added and incubated at room temperature for 30 min with the samples. Then the sections were subsequently treated with a secondary antibody [HRP-labeled goat resistant rabbit IgG; 1:1,000; cat. no. abs957; Absin (Shanghai) Biotechnology Co., Ltd.] at room temperature for $1 \mathrm{~h}$. The slices were stained with 3',3'-diaminobenzidine (DAB) at room temperature for about $7 \mathrm{~min}$ [Liquid $\mathrm{A}$ and liquid $\mathrm{B}$; 1000:50; cat. no. abs957; Absin (Shanghai) Biotechnology Co., Ltd.]. Finally, the slides were stained with $0.02 \%$ hematoxylin (cat. no. BL702A; Biosharp Life Sciences) and observed under a light microscope (IX73; Olympus Corporation). The images were analyzed using Photoshop (Adobe Systems, Inc.).

A total of two pathologists from The Affiliated Hospital of Xuzhou Medical University (Xuzhou, China) examined the pathological sections separately under blinded experimental conditions and all the differences were evaluated by discussion. High power microscopic fields (two), that were representative of the samples were selected for each specimen and 200 tumor cells were counted in each field. The German IHC Score (GIS) (11) was used for scoring. The intensity of ARL2 immunostaining was scored as follows: 0, negative; 1, weak; 2, moderate; 3 , strong, whereas the percentage of immunoreactive cells was categorized as 1 (0-25\%), 2 (26-50\%), 3 (51-75\%), and 4 (76-100\%). The IHC expression values of ARL2 and the associated clinical information of each sample are shown in Table II.

Cell lines and culture conditions. The HCT8, HCT116 and 293T cell lines were acquired from the Cancer Institute, Xuzhou Medical University (Jiangsu, China), which were originally purchased from the Shanghai Institute of Biochemistry and Cell Biology, Chinese Academy of Science (Shanghai, China). The HCT8, HCT116 and 293T cell lines were authenticated using short tandem repeat DNA profiling, and subsequently cultured in DMEM containing $10 \%$ fetal bovine serum (cat. no. SH30022.01B; HyClone; Cytiva), $100 \mu \mathrm{g} / \mathrm{ml}$ streptomycin and $100 \mathrm{U} / \mathrm{ml}$ penicillin, and incubated at $37^{\circ} \mathrm{C}$ in a humidified incubator with $5 \% \mathrm{CO}_{2}$.

RNA isolation and reverse transcription-quantitative PCR $(R T-q P C R)$. Total RNA was extracted from the HCT8 and HCT116 cells using TRIzol ${ }^{\circledR}$ (Invitrogen; Thermo Fisher Scientific, Inc.), in line with the manufacturer's protocol. The total RNA was reverse transcribed into cDNA for PCR amplification. RT-qPCR was performed using TransStart ${ }^{\circledR}$ Top Green qPCR SuperMix Assays (cat. no. AQ131; Beijing Transgen Biotech Co., Ltd.) and in a thermal cycler (LightCycler ${ }^{\circledR} 480 \mathrm{II}$; Roche Diagnostics). Human mRNA sequences, encoding ARL2 and AXL genes, were retrieved from the nucleotide database of National Center for Biotechnology Information (NCBI, https://www.ncbi.nlm.nih.gov/). The primers were designed using Primer Premier v5.0 (Premier Biosoft) and Basic Local Alignment Search Tool was used in NCBI to determine the specificity of the primers. The primers were synthesized by Generay Biotech Co., Ltd. The following thermocycling conditions were used: Initial denaturation at $95^{\circ} \mathrm{C}$ for $30 \mathrm{sec}$, followed by 40 cycles of a two-step cycling program $\left(95^{\circ} \mathrm{C}\right.$ for $5 \mathrm{sec}$ and $60^{\circ} \mathrm{C}$ for $\left.30 \mathrm{sec}\right)$. The mRNA expression was normalized to the expression of $\beta$-actin and calculated using the $2^{-\Delta \Delta \mathrm{Cq}}$ method (19). The specific primers for ARL2, AXL and $\beta$-actin were as follows: ARL2 forward, 5'-GGGAGGACATCGACACCA-3' and reverse, 5'-AGGACC GCAGGGACTTC-3'; AXL forward, 5'-GTTTGGAGCTGT 
Table I. Clinical characteristics of the 19 patients with colorectal cancer.

\begin{tabular}{lr}
\hline Characteristics & Number of patients $(\%)$ \\
\hline Age, years & \\
$<60$ & $5(26.32)$ \\
$\geq 60$ & $14(73.68)$ \\
Sex & \\
Male & $7(36.84)$ \\
Female & $12(63.16)$ \\
Cancer location & \\
Proximal & $11(57.89)$ \\
Distal colon & $8(42.11)$ \\
Degree of differentiation & \\
Well & $6(31.58)$ \\
Moderately & $6(31.58)$ \\
Poorly & $7(36.84)$ \\
\hline
\end{tabular}

Table II. ARL2 expression value and characteristics of the normal healthy and CRC samples used for IHC.

\begin{tabular}{|c|c|c|c|}
\hline Sample & $\begin{array}{l}\text { Sample } \\
\text { number }\end{array}$ & Age, years & GIS \\
\hline \multirow[t]{3}{*}{ Normal healthy tissue } & N1 & 35 & 0 \\
\hline & N2 & 60 & 1 \\
\hline & N3 & 85 & 1 \\
\hline \multicolumn{4}{|l|}{ CRC tissues } \\
\hline \multirow[t]{6}{*}{ Well differentiated } & H1 & 66 & 8 \\
\hline & $\mathrm{H} 2$ & 63 & 12 \\
\hline & $\mathrm{H} 3$ & 85 & 12 \\
\hline & $\mathrm{H} 4$ & 40 & 8 \\
\hline & H5 & 67 & 9 \\
\hline & H6 & 62 & 12 \\
\hline \multirow[t]{6}{*}{ Moderately differentiated } & M1 & 66 & 4 \\
\hline & M2 & 80 & 8 \\
\hline & M3 & 82 & 8 \\
\hline & M4 & 50 & 4 \\
\hline & M5 & 78 & 4 \\
\hline & M6 & 54 & 8 \\
\hline \multirow[t]{7}{*}{ Poorly differentiated } & L1 & 73 & 4 \\
\hline & $\mathrm{L} 2$ & 66 & 4 \\
\hline & L3 & 37 & 0 \\
\hline & L4 & 76 & 0 \\
\hline & L5 & 55 & 6 \\
\hline & L6 & 70 & 8 \\
\hline & L7 & 77 & 8 \\
\hline
\end{tabular}

IHC, immunohistochemistry; GIS, German IHC Score.
CCGCAAAG-3' and reverse, 5'-CTGGAAGGTGGACAG CGAGG-3'.

Immunofluorescence. ARL2 pcDNA3.1 HCT8 and HCT116 cells, ARL2 shRNA HCT8 and HCT116 cells, and the corresponding control group cells were fixed with $4 \%$ paraformaldehyde at room temperature for $20 \mathrm{~min}$ and blocked in PBS containing 5\% goat serum [cat. no. abs933; Absin (Shanghai) Biotechnology Co., Ltd.]. The cells were then washed three times, with PBS for 5 min and permeabilized with $0.3 \%$ Triton X-100 for $1 \mathrm{~h}$. An additional washing step with PBS was performed three times, for 5 min each time, then 5\% BSA (cat. no. EPR12774; Abcam, Inc.) was incubated with the cells for $1 \mathrm{~h}$ at room temperature. The samples were incubated overnight at $4^{\circ} \mathrm{C}$ with a primary antibody against ARL2 (cat. no. 188322; 1:100; Cell Signaling Technology, Inc.). The samples were subsequently washed with PBS three times for $5 \mathrm{~min}$ each time, then they were incubated with the secondary antibody (goat polyclonal secondary antibody against rabbit IgG-H\&L, Alexa Fluor ${ }^{\circledR} 647$; cat. no. ab150083; Abcam). An Olympus IX73 microscope was used to capture the fluorescence images (Olympus Corporation). For the quantitative analysis of the intensity of fluorescence expression, the immunofluorescence pictures were quantitatively analyzed using Image-Pro Plus 1.8.0 software (Media Cybernetics Inc.) to calculate the mean gray value of the represented area in each picture.

Western blot analysis. Western blot analysis was performed, as previously described (20). ARL2 and AXL were resolved on 12 and 6\% SDS-PAGE, respectively, then transferred to nitrocellulose membranes. Primary antibodies against the following proteins were used: ARL2 (cat. no. 188322; Cell Signaling Technology, Inc.), AXL (cat. no. 8661; Cell Signaling Technology, Inc.), $\beta$-actin (cat. no. 66009-1-Ig; ProteinTech Group, Inc.). The following secondary antibodies were used: HRP-goat anti-mouse $(1: 2,000$; cat. no. SA00004-1; Proteintech Inc.) and HRP-goat anti-rabbit (1:2,000; cat. no. SA00004-2; Proteintech Inc.). A Tanon 5200 automatic chemiluminescence imaging analysis system was used to detect the protein bands with an enhanced chemiluminescence reagent (both from Tanon Science and Technology Co., Ltd.). For the quantitative analysis of the intensity of protein expression, the pictures were quantitatively analyzed using Image J v.1.8.0 software (National Institutes of Health Inc.) to calculate the expression levels of protein bands.

Plasmids and viral infection. The vectors ARL2 pcDNA3.1-3xFlag-C (pcDNA3.1) and ARL2 pLVX-short hairpin (sh) RNA-PURO (shRNA) were purchased from YouBio Technology Co. Ltd. The ARL2 pcDNA3.1 was transfected into the HCT8 and HCT116 cells, with the two packing vectors, pMD2G and psPAX (Shanghai YouBio Technology Co., Ltd.), as previously described (21). Stable cell lines overexpressing ARL2 were selected following treatment with $40 \mu \mathrm{g} / \mathrm{ml} \mathrm{G} 418$ (antibiotic) for $\sim 2$ weeks. The ARL2 interfering viruses were generated by co-transfecting $293 \mathrm{~T}$ cells with the other two packing vectors (pMD2G and psPAX), and concentrated as previously described (22). Stable ARL2 
A
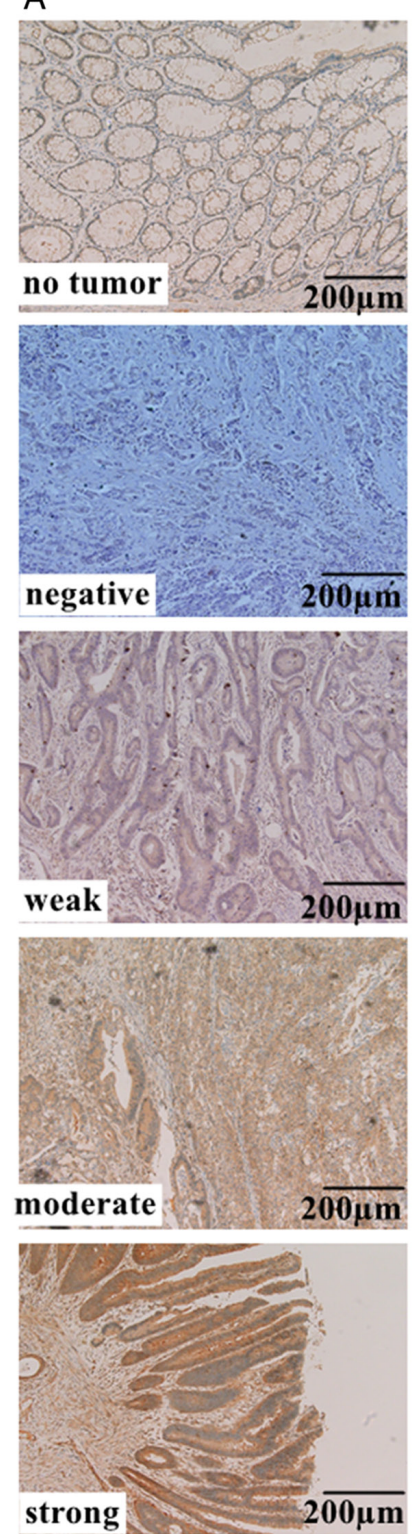
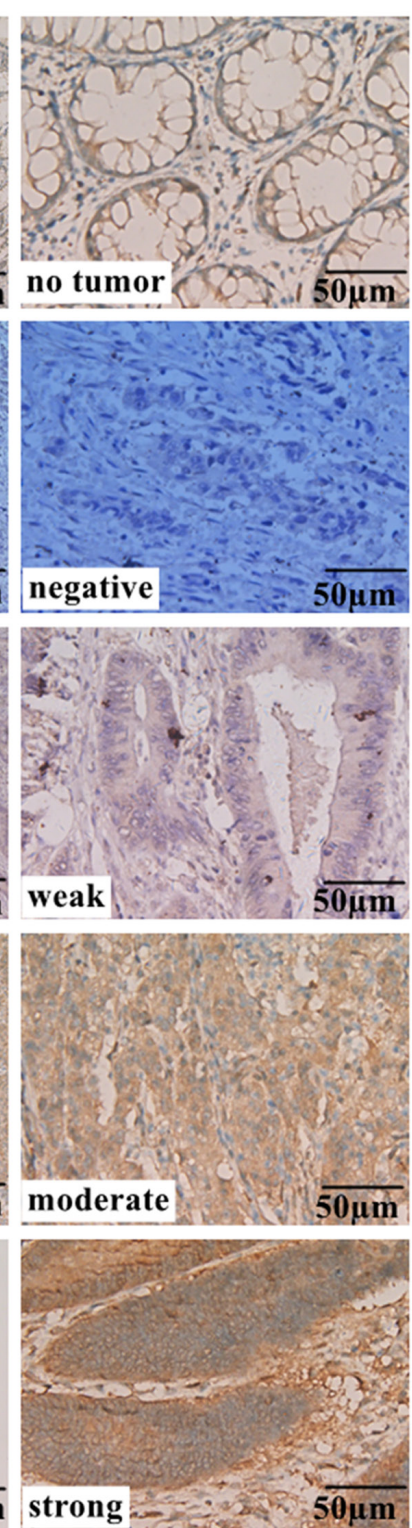

B

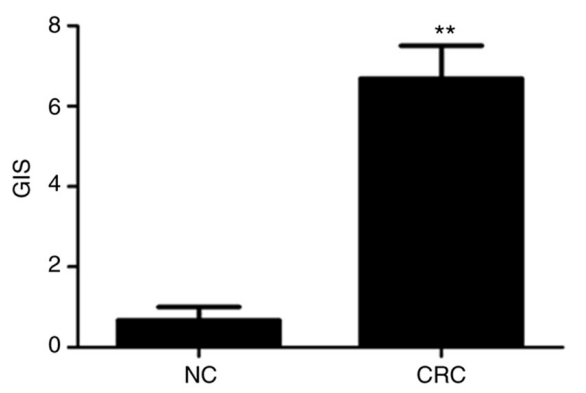

C

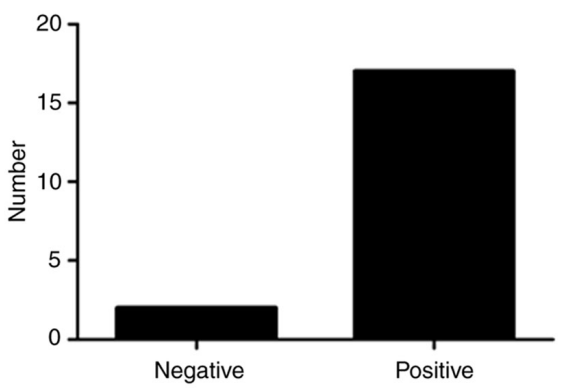

D

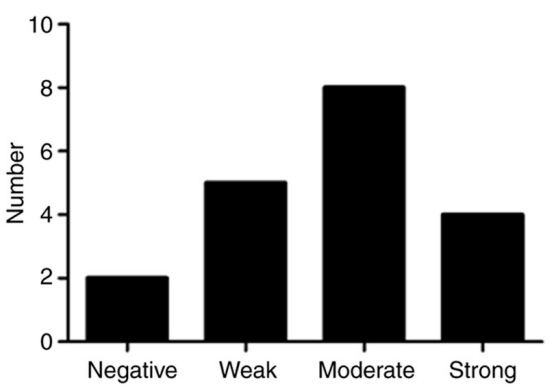

Figure 1. ARL2 protein expression levels are increased in patients with CRC tumor tissues. (A) Representative immunohistochemistry images of the ARL2 protein expression in normal healthy $(n=3)$ and colon cancer samples CRC $(n=19)$. Magnification of the images is $x 10$ and 40 in the left and right panels respectively. (B) Analysis of GIS between NC and CRC groups. (C) Distribution of ARL2 negative and positive expression levels in CRC tissues. (D) Distribution of ARL2 expression level using GIS in CRC tissues. ${ }^{* *} \mathrm{P}<0.001$. IHC, immunohistochemistry; ARL2, ADP ribosylation factor-like GTPase 2; CRC, colorectal cancer; NC, normal control; GIS, German IHC Score.

knockdown cell lines were generated by infection of the cells with lentivirus and clone selection with $2 \mu \mathrm{g} / \mathrm{ml}$ puromycin for $\sim 2$ weeks.

Cell proliferation and colony formation. The Cell Counting Kit (CCK)-8 method was used to detect cell proliferation according to the manufacturer's protocol (cat. no. VC5001; VICMED Life Sciences). The in vitro cell proliferation assay method was performed by diluting $5 \times 10^{3}$ cells in $100 \mu 1$ medium. The ARL2 pcDNA3.1 HCT8 and HCT116 cells, ARL2 shRNA HCT8 and HCT116 cells, and the corresponding control group cells were transferred into 96-well plates and incubated at $37^{\circ} \mathrm{C}$ with $5 \% \mathrm{CO}_{2}$ for 6 days. On days $0,1,2$, 3 and $4,10 \mu 1$ CCK- 8 was added to the wells and incubated for $4 \mathrm{~h}$ at $37^{\circ} \mathrm{C}$ to detect cell proliferation. Subsequently, the optical density (OD) of each well was measured using a microplate reader (Tecan Spark 84669JK7573; Tecan Group, Ltd.) at $450 \mathrm{~nm}$. The growth curve was created according to the OD of each well. Following ARL2 overexpression or knockdown, the colony formation assay was performed. The cells were collected, resuspended as single cells $\left(0.5 \times 10^{5}\right)$ and seeded into a 6-well plate, which was incubated at $37^{\circ} \mathrm{C}$ with $5 \% \mathrm{CO}_{2}$. Following 2 weeks of incubation, the cells were washed with PBS twice and stained with crystal violet solution at room temperature for $\sim 20 \mathrm{~min}$. The number of colonies ( $>50$ cells) were measured under a light microscope (IX73; Olympus Corporation).

Wound healing assay. ARL2 pcDNA3.1 HCT8 and HCT116 as well as ARL2 shRNA HCT8 and HCT116 cells (5x10 5 per well) 
A
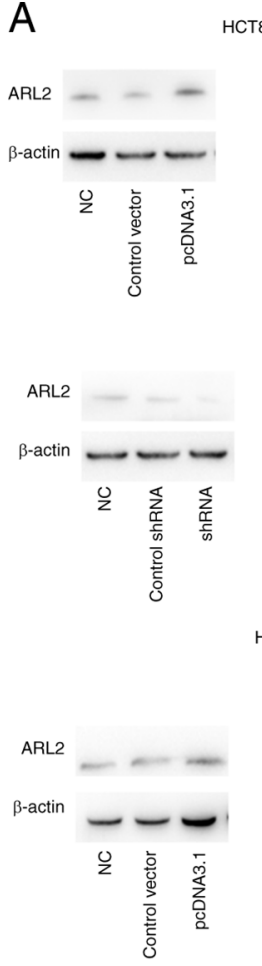

HCT116
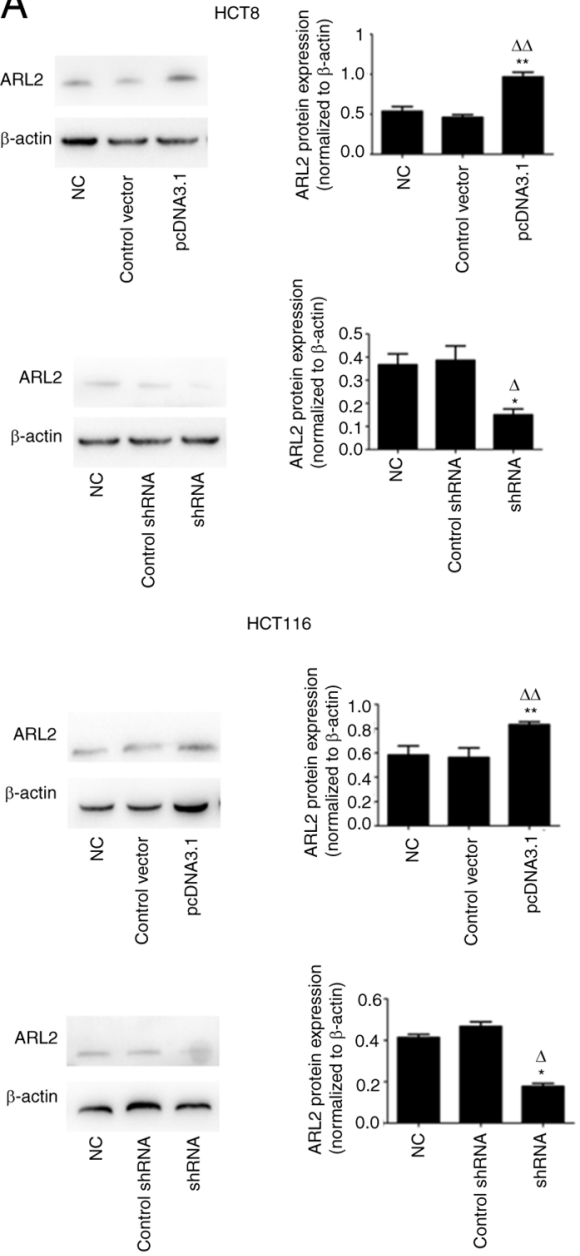

B

Control vector
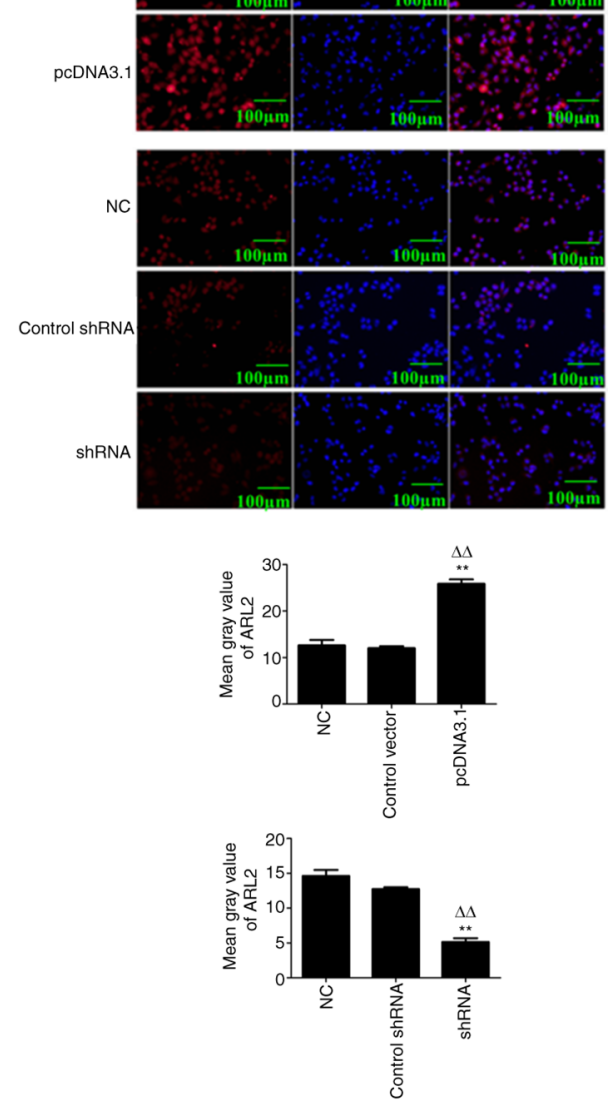
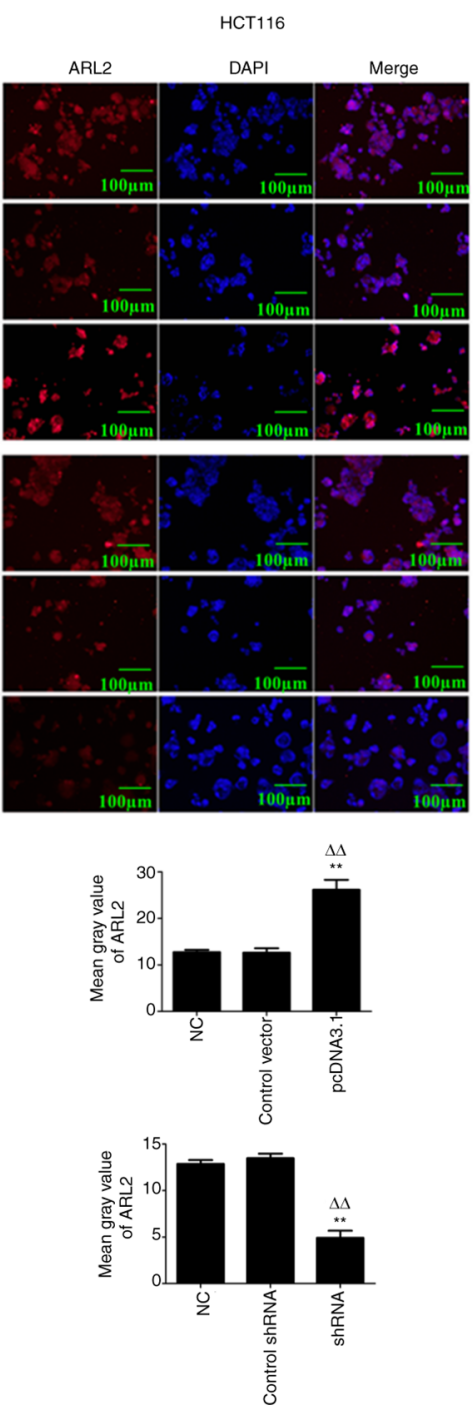

Figure 2. Generation of pcDNA3.1 and shRNA in the HCT8 and HCT116 cell lines. (A) Representative western blot images and analysis of ARL2 protein expression level in the HCT8 and HCT116 cells transfected with pcDNA3.1 or shRNA or their respective controls ( $\mathrm{n}=3$; ANOVA). "P<0.05 vs. NC group; ${ }^{* *} \mathrm{P}<0.001$ vs. $\mathrm{NC}$ group; ${ }^{\Delta} \mathrm{P}<0.05$ vs. control vector; ${ }^{\Delta \Lambda} \mathrm{P}<0.001$ vs. control vector. (B) The representative immunofluorescence images of ARL2 expression in HCT8 and HCT116 cells transfected with pcDNA3.1 and shRNA or their corresponding control vectors. Magnification, x20. Scale bar, $100 \mu \mathrm{m}$. NC, normal control; ARL2, ADP ribosylation factor-like GTPase 2; sh, short inhibiting.

were incubated in 6-well plates for $24 \mathrm{~h}$. When the cells were $\sim 100 \%$ confluent, the monolayer was scratched with a $200 \mu \mathrm{l}$ pipette tip. The suspension cells were washed with PBS and fresh serum-free medium was added. The cells were cultured at $37^{\circ} \mathrm{C}$ with $5 \% \mathrm{CO}_{2}$ and the wound was allowed to heal. The images of the wound were captured at 0 and $24 \mathrm{~h}$. The wound closure rates were measured based on previous reports (11). The ImageJ software (v.1.48, National Institutes of Health) was used to evaluate the wound area, and the wound closure rate was calculated using the following formula: (original wound area/actual wound area)/area of the original wound $\mathrm{x} 100$.

Matrigel transwell invasion assay. The assay was performed using an $8-\mu \mathrm{m}$ filter membrane (cat. no. 3422; Corning Inc.). A total of $100 \mu 1$ Matrigel (cat. no. 356234; Corning Inc.) (diluted with serum-free DMEM by 1:8) was plated in the Transwell chamber and kept in an incubator at room temperature. Following $4 \mathrm{~h}$ of incubation, $200 \mu \mathrm{l}$ serum-free medium containing $1 \times 10^{5}$ cells was added to the upper chamber of the Transwell insert, while $750 \mu$ l DMEM with $10 \%$ FBS (cat. no. 26140079; Gibco; Thermo Fisher Scientific Inc.) was added to the lower chamber. The cells were incubated at $37^{\circ} \mathrm{C}$ in $5 \% \mathrm{CO}_{2}$ for $16 \mathrm{~h}$. A cotton swab was used to remove the apical matrix and the cells. Following fixation with $4 \%$ paraformaldehyde at room temperature for $\sim 10 \mathrm{~min}$, the transmembrane cells were stained with crystal violet staining solution at room temperature for $\sim 20 \mathrm{~min}$, and each well was counted at 5 different high-power microscope fields under a light microscope (magnification, x400). The experiment was conducted in triplicate.

Statistical analysis. All the experiments were performed independently, three times. The data are presented as the mean \pm SD. The number of repetitions for each experiment is illustrated in the corresponding figure. Statistical differences between two groups were determined using a two tailed t-test, and the differences among $>2$ groups were analyzed one-way ANOVA followed by the Dunnett's post-test. SPSS v20.0 
A
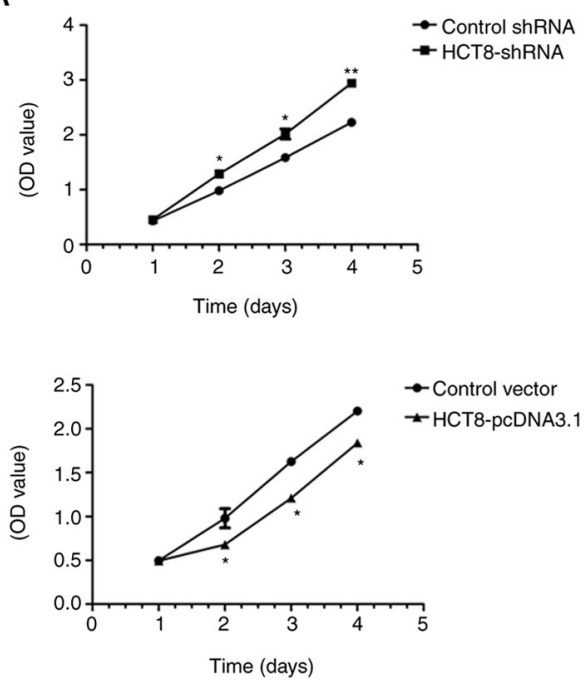

C
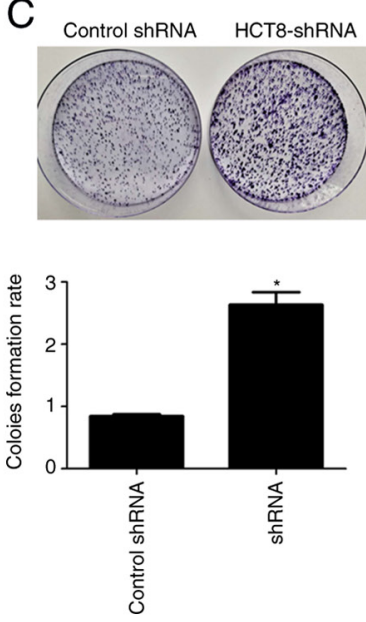

Control vector HCT8-pcDNA3.1
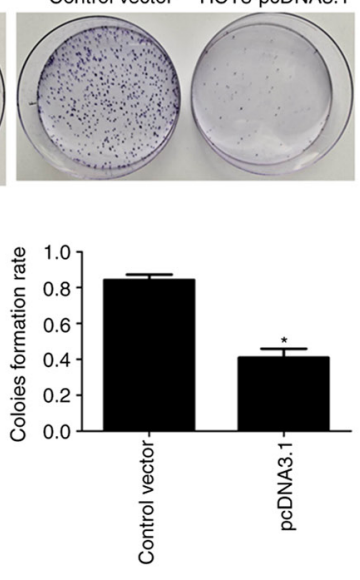

B

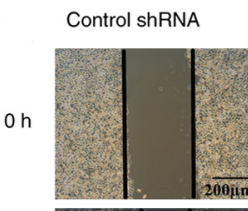

HCT8-shRNA

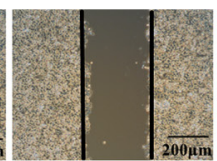

Control vector

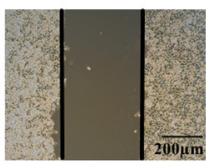

HCT8-pcDNA3.1
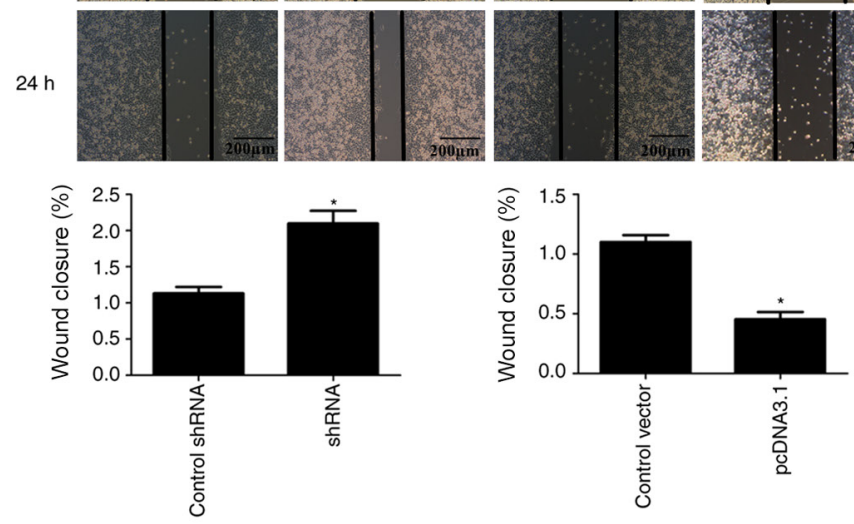

D

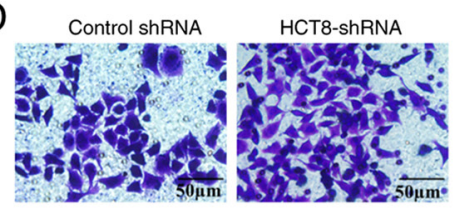

Control vector
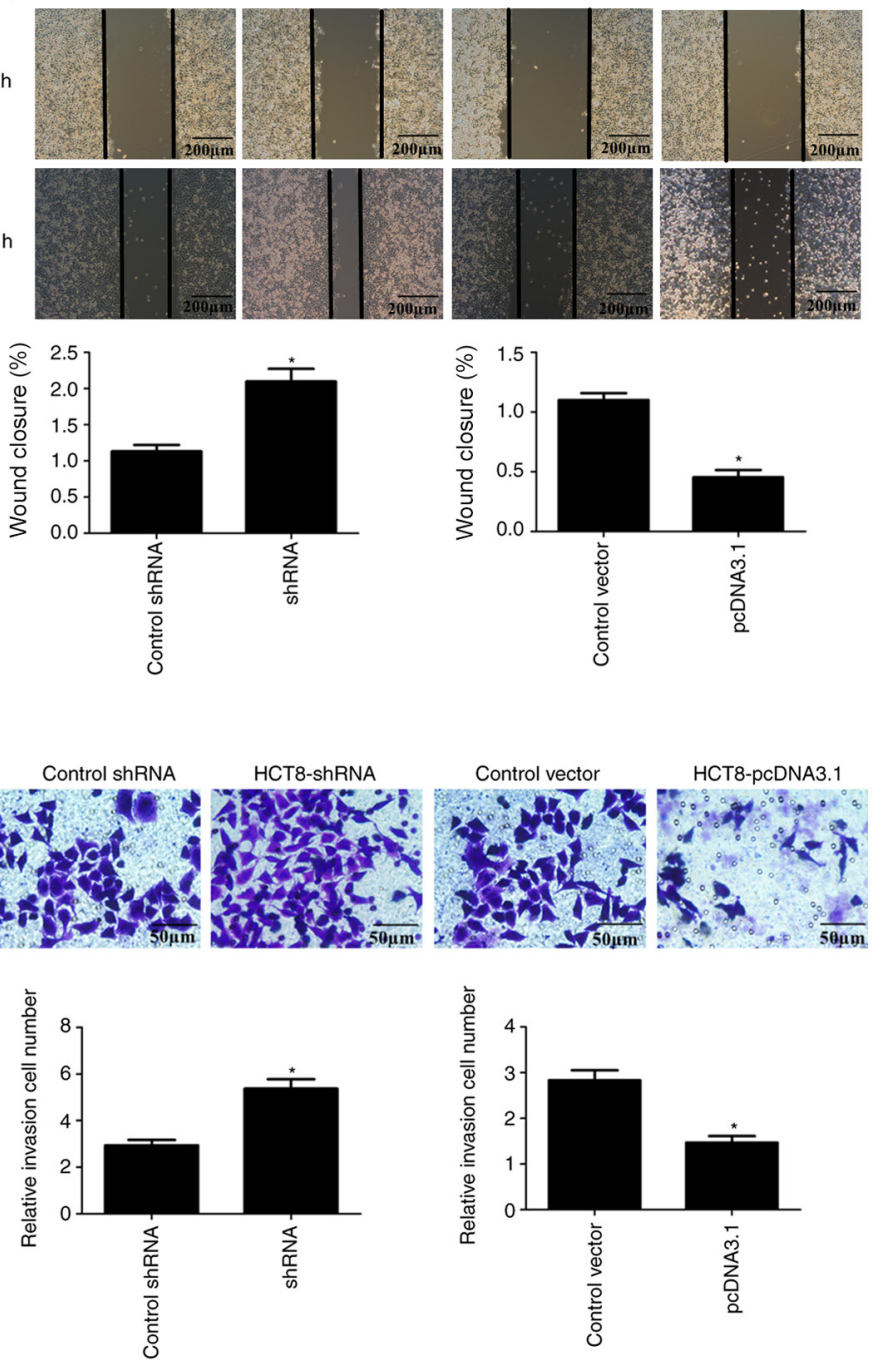

Figure 3. ARL2 expression and HCT8 proliferation, migration and invasion. (A) Cell Counting Kit-8 assays were used to examine HCT8 cell proliferation in control and ARL2 overexpressing and knockdown HCT8 cells. (B) Wound healing assays were used to examine cell migration in control and ARL2 overexpressing and knockdown HCT8 cells. Scale bar, $200 \mu \mathrm{m}$. (C) Colony formation assays were used to examine HCT8 cell proliferation in control and ARL2 overexpressing and knockdown HCT8 cells. (D) Matrigel assay was used to examine cell invasion in control, ARL2 overexpressing and knockdown HCT8 cells. Scale bar, $50 \mu \mathrm{m}$. The data are presented as the mean $\pm \mathrm{SD}$ from three independent experiments performed in triplicate. ${ }^{*} \mathrm{P}<0.05 \mathrm{vs}$. control vector or control shRNA, ${ }^{* *} \mathrm{P}<0.001$ vs. control vector or control shRNA. ARL2, ADP ribosylation factor-like GTPase 2; sh, short inhibiting; pcDNA3.1, overexpressing ARL2 plasmid; OD, optical density.

(IBM, Corp.) and GraphPad Prism v6.0 (GraphPad Software, Inc.) were used for statistical analysis. $\mathrm{P}<0.05$ was considered to indicate a statistically significant difference.

\section{Results}

Expression of ARL2 is increased in colon cancer tissues. A total of 22 human clinical specimens were collected, including $19 \mathrm{CRC}$ tissues (6 well-differentiated, 6 moderately-differentiated and 7 poorly differentiation cases) and 3 normal healthy colon tissue. In 17 of the 19 specimens (89.47\%) (Fig. 1C), ARL2 was expressed in $\geq 1 \%$ cancer cells, with different staining intensities: 5 with weak staining, 8 with moderate staining, and 4 with strong staining (Fig. 1A and D). There were significant differences in the expression level of ARL2 between CRC and normal healthy colon tissue (Fig. 1B).
Taken together, these results demonstrated that the protein expression levels of ARL2 were significantly increased in CRC.

Overexpressed ARL2 decreases proliferation and colony-formation abilities of CRC cells. To investigate the physiological function of ARL2 in colon cancer, ARL2 overexpressing (pcDNA3.1) and knockdown (shRNA) cell lines were established using plasmid transfection and viral infection in 2 colon cancer cell lines (HCT8 and HCT116). Western blot analysis indicated that the protein expression levels of ARL2 were significantly increased or decreased, respectively following transfection with overexpression or knockdown plasmids in both the cell lines (Fig. 2A; $\mathrm{P}<0.05$ ). Furthermore, immunofluorescence confirmed that ARL2 protein expression level was significantly increased or reduced following transfection with overexpression or knockdown plasmids, respectively, in both the cell lines (Fig. 2B). 
A
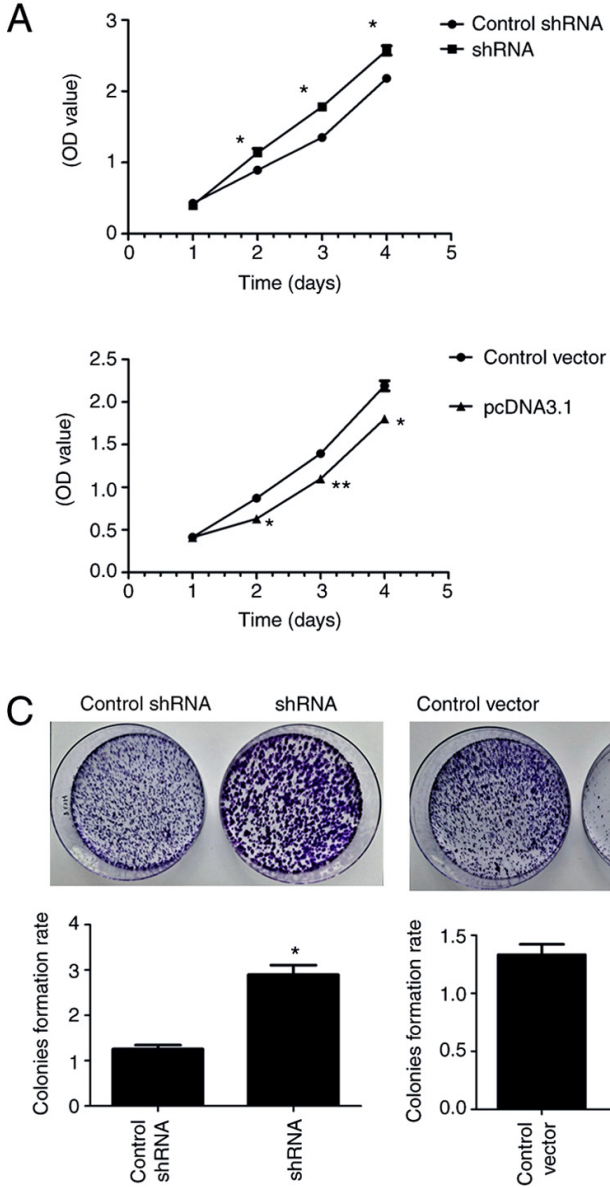
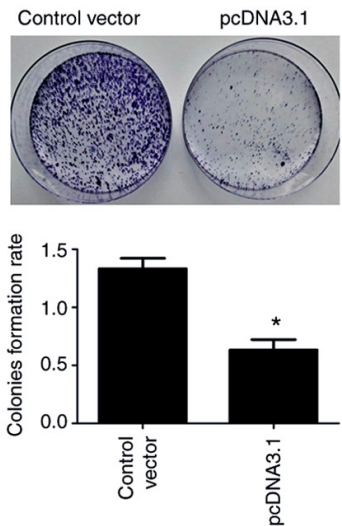

B
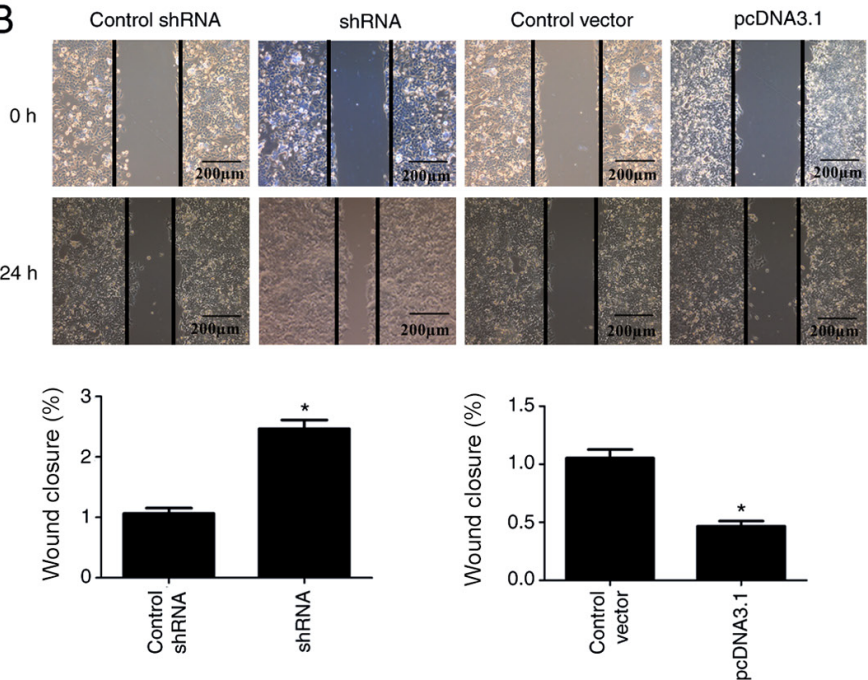

$\mathrm{D}$
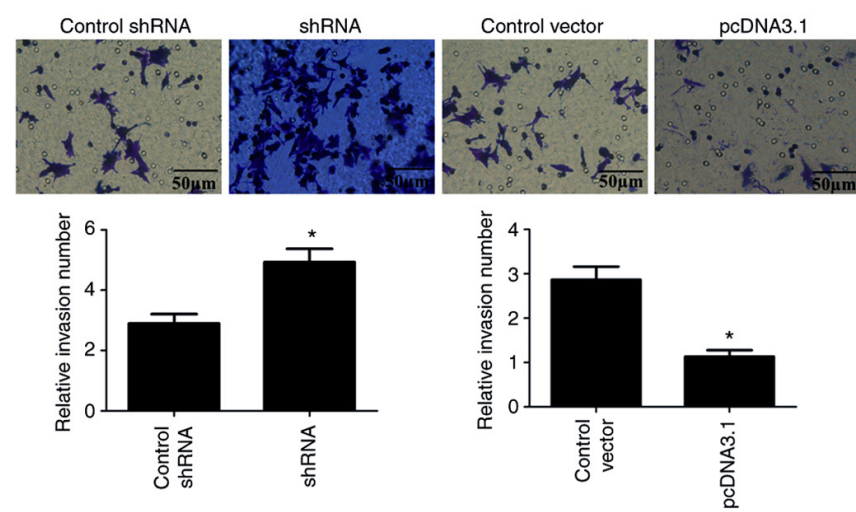

Figure 4. ARL2 expression and HCT116 proliferation, migration and invasion. (A) Cell Counting Kit-8 assays were used to examine HCT116 cell proliferation in control, ARL2 overexpressing and knockdown HCT8 cells. (B) Wound healing assays were used to examine cell migration in control, ARL2 overexpressing and knockdown HCT116 cells. Scale bar, $200 \mu \mathrm{m}$. (C) Colony formation assays were used to examine HCT116 cell proliferation in control, ARL2 overexpressing and knockdown HCT116 cells. (D) Matrigel assay was used to examine cell invasion in control, ARL2 overexpressing and knockdown HCT116 cells Scale bar, $50 \mu \mathrm{m}$. The data are presented as the mean \pm SD from three independent experiments performed in triplicate. * $\mathrm{P}<0.05$ vs control vector or control shRNA, ${ }^{* *} \mathrm{P}<0.001$ vs. control vector or control shRNA. ARL2, ADP ribosylation factor-like GTPase 2; sh, short inhibiting; pcDNA3.1, overexpressing ARL2 plasmid; OD, optical density.

The data indicated that overexpression of ARL2 (pcDNA3.1) significantly inhibited the proliferation of the HCT8 and HCT116 cells, while ARL2 knockdown (shRNA) significantly increased the proliferation of the HCT8 (Fig. 3A) and HCT116 (Fig. 4A) cells $(\mathrm{P}<0.05)$.

In addition, the colony formation assay was performed to detect the colony-formation ability of the CRC cells. As expected, colony formation of the HCT8 and HCT116 cells, with ARL2 overexpression plasmid, was significantly decreased compared with that in the control group. In contrast, the HCT8 and HCT116 cells with ARL2 shRNA sequences demonstrated significantly increased colony formation ability compared with that in the control group (Figs. $3 \mathrm{C}$ and $4 \mathrm{C}$; $\mathrm{P}<0.05)$. These data indicated that ARL2 reduced the proliferation of the HCT8 and HCT116 cells.

Overexpressed ARL2 inhibits the migratory and invasive abilities of the CRC cells. The microtubule network has a critical role in regulating cell migration and invasion (23); therefore, the wound healing and Matrigel assays were used to examine whether overexpression or ARL2 knockdown inhibited or promoted the migration and invasion of the CRC cells. As shown in Figs. 3B and 4B, the migration ability of the HCT8 and HCT116 cells, with ARL2 overexpressing plasmid, was significantly decreased compared with that in the control group, while the migration ability of the HCT8 and HCT116 cells, with ARL2 knockdown plasmid was significantly increased compared with that in the control group.

The Matrigel assay revealed similar results demonstrating that ARL2 overexpressing cells (pcDNA3.1) had reduced invasive abilities, whereas ARL2 knockdown (shRNA) cells exhibited increased invasive abilities (Figs. 3D and 4D).

These results indicated that ARL2 reduced the migratory and invasive abilities of the HCT8 and HCT116 cells.

$A R L 2$ reduces $A X L$ expression in the CRC cells. To investigate the functions of ARL2 in CRC, its downstream target, AXL (11) was studied in additional experiments. Previous studies confirmed that AXL played a key role in the functional regulation of CRC cells $(16,17)$. On this basis, the effects of ARL2 expression on AXL were investigated in the HCT8 and HCT116 cells. Initially, western blot analysis was used to detect the expression levels of AXL in the HCT8 and HCT116 cells, following ARL2 overexpression and knockdown. The 


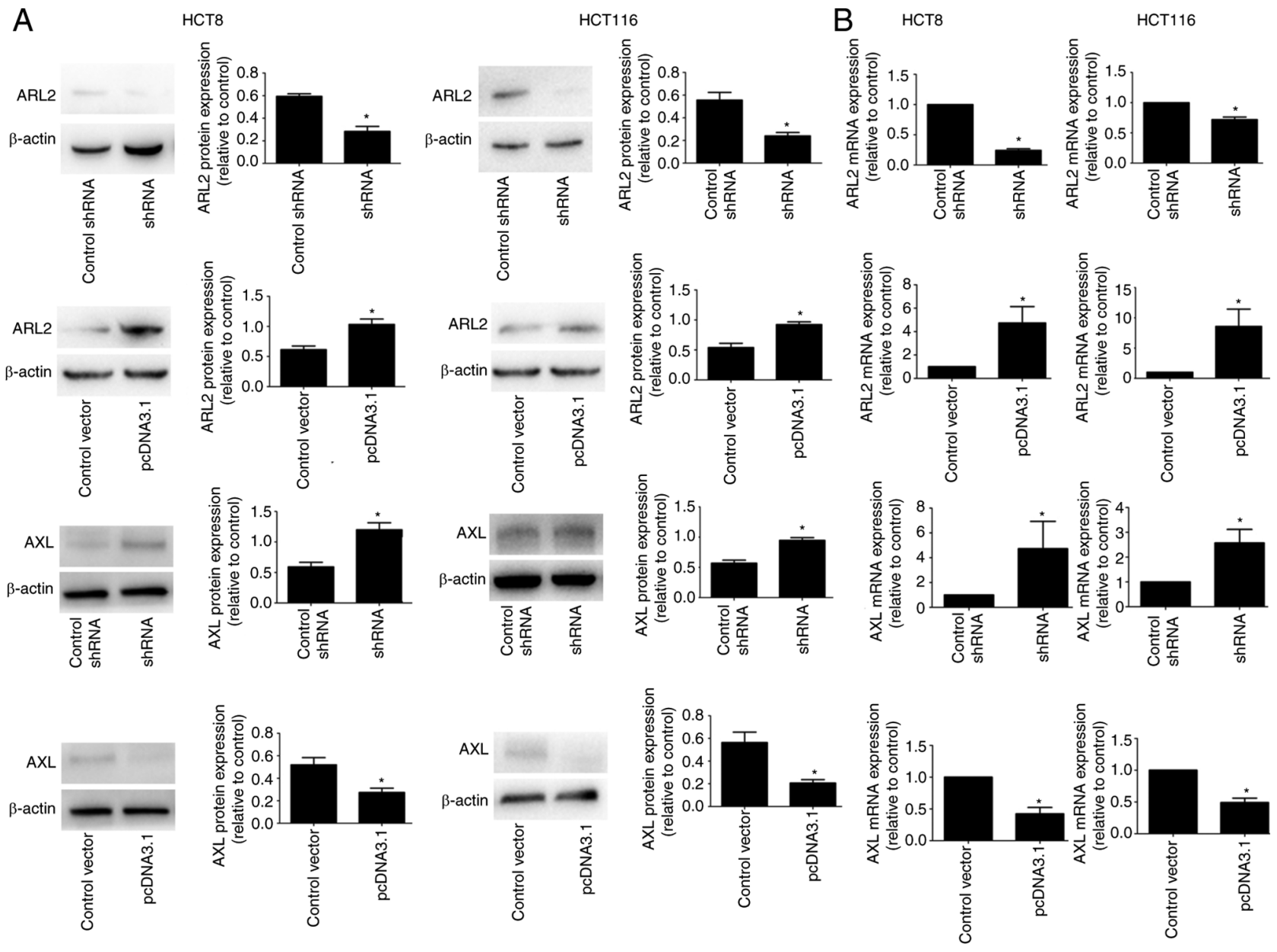

Figure 5. ARL2 and AXL protein and mRNA expression levels. (A) Western blot analysis was performed in ARL2 overexpression and knockdown HCT8 and HCT116 cells for the detection of AXL and ARL2 protein expression level. (B) Reverse transcriptions-quantitative PCR was performed to analyze ARL2 and AXL mRNA expression levels in HCT8 and HCT116 cells, following transfection with ARL2 overexpression and knockdown vectors. The data are presented as the mean \pm SD from three independent experiments performed in triplicate. ${ }^{*} \mathrm{P}<0.05$ vs. control vector. ARL2, ADP ribosylation factor-like GTPase 2 ; sh, short inhibiting; pcDNA3.1, overexpressing ARL2 plasmid.

results indicated that ARL2 overexpression reduced AXL protein expression, whereas ARL2 knockdown increased AXL protein expression levels (Fig. 5A). In addition, RT-qPCR assays demonstrated that ARL2 overexpression decreased AXL mRNA expression level, whereas ARL2 knockdown increased AXL mRNA expression level (Fig. 5B).

With increased ARL2 protein and mRNA expression levels, there was a decrease in AXL protein and mRNA expression levels, while the opposite results were found when ARL2 expression was knocked down.

\section{Discussion}

Microtubule network dynamics is important to the regulation of physiological processes, such as cell mitosis and migration (24,25). For example, ARL2 has been associated with the occurrence of specific malignant tumors, such as glioma, breast cancer and cervical cancer $(11,13,15)$. However, the protein expression pattern and pathophysiological role of ARL2 in different types of cancer is controversial, as the expression of ARL2 is decreased in glioma and increased in hepatocellular carcinoma $(11,14)$, and the role of ARL2 in CRC is unclear. In the present study, increased protein expression levels of ARL2 were found in CRC tissues, which is consistent with a previous study (26). ARL2 overexpression suppressed migration, invasion and proliferation of two CRC cell lines (HCT8 and HCT116). In contrast, ARL2 knockdown enhanced migration, invasion and proliferation. There was decreased AXL expression in the CRC cells when ARL2 was overexpressed. The results of the present study suggested that ARL2 may regulate the migration and invasion of the CRC cells by negatively regulating AXL expression. Therefore, ARL2 may be considered a protective factor against CRC development.

An increasing number of studies have demonstrated that ARL2 was associated with the development and progression of various types of cancer. ARL2 has been reported to inhibit the proliferation, migration and tumorigenicity of glioma cells and may be considered a new prognostic and therapeutic target for glioma (11). It was also reported that ARL2 played an oncogenic role in cervical cancer (15). The current study demonstrated that ARL2 protein expression level was increased in CRC tissues, while its overexpression decreased cell migration, invasion and proliferation, suggesting that it may be a protective factor against CRC progression. 
As a member of the TAM group of receptors, AXL acts as an inhibitor of cytokine receptors mediates cell activation and promotes apoptotic cell removal $(27,28)$. AXL gene silencing and drug inhibition suppressed proliferation, migration and survival in CRC cells $(16,17)$. In the present study, the results indicated that ARL2 was inversely associated with AXL expression in CRC cells. Furthermore, previous studies have indicated that AXL functions as a tumor promoter in cancer. For example, AXL induced the activation of downstream AKT, and inhibited the proliferation and migration of pancreatic cancer cells in vitro (29). In addition, GAS6 promoted gastric cancer invasiveness by activating AXL (30). Previous studies have demonstrated the role of $\mathrm{AXL}$ in regulating cell growth, migration and tumorigenesis of CRC cells $(29,30)$. AXL acted as a tumor promoter $(31,32)$, which may partially be suppressed by overexpressed ARL2 expression in CRC cells. The present study provided the first preliminary evidence of AXL was negatively associated with ARL2. To elucidate the mechanism by which ARL2 and AXL expression in CRC cells, western blot and RT-qPCR analyses were used to detect the protein and mRNA expression levels of ARL2 and AXL in HCT8 and HCT116 cells, following overexpression or knockdown of ARL2. At both the protein and mRNA expression, increased ARL2 led to decreased AXL and decreased ARL2 led to increased AXL. The results revealed that the increase in the levels of ARL2 was accompanied by decrease in AXL expression level and may have reduced CRC cell progression. Therefore, ARL2 may be negatively correlated with the expression level of AXL at the gene level.

In conclusion, the present study described the increased expression levels of ARL2 in clinical CRC samples. ARL2 could play a crucial role in CRC. In addition, significant evidence was provided demonstrating that increased ARL2 expression in CRC cells inhibited proliferation, colony formation, migration and invasion. Furthermore, the results confirmed that ARL2 was negatively associated with AXL in CRC cells and maybe a protective factor in CRC. Taken together, the results suggested that ARL2 could play an important inhibitory role in the proliferation, migration and tumorigenicity of CRC cells and ARL2 was negatively associated with AXL expression. Therefore, ARL2 may be a new predictive and therapeutic target for CRC.

\section{Acknowledgements}

The authors would like to thank Dr Yingying Cui and Dr Kai Cao (Department of Pathology, The Affiliated Hospital of Xuzhou Medical University) for discussions pertaining to the present study.

\section{Funding}

No funding was received.

\section{Availability of data and materials}

The datasets used and/or analyzed in the current study are available from the corresponding author upon reasonable request.

\section{Authors' contributions}

SF designed the study. XP, YW, BM and WC performed the experiments and analyzed the data. WC, YW and XP wrote the manuscript. SF and BM revised the manuscript. All authors read and approved the final version of the manuscript and agree to be accountable all aspects of the study to ensure the accuracy or integrity of all parts of the work.

\section{Ethics approval and consent to participate}

This study was approved by the Institutional Ethics Committee of the Affiliated Hospital of Xuzhou Medical University (approval no. XYFY2019-KL157-01) and adhered to the principles of the Declaration of Helsinki. Each patient provided written informed consent.

\section{Patient consent for publication}

Not applicable.

\section{Competing interests}

The authors declare that they have no competing interests.

\section{References}

1. Mattiuzzi C, Sanchis-Gomar F and Lippi G: Concise update on colorectal cancer epidemiology. Ann Transl Med 7: 609-609, 2019.

2. Gu X, Zheng R, Xia C, Zeng H, Zhang S, Zou X, Yang Z, Li H and Chen W: Interactions between life expectancy and the incidence and mortality rates of cancer in China: A population-based cluster analysis. Cancer Commun (Lond) 38: 44, 2018.

3. Zeng H, Chen W, Zheng R, Zhang S, Ji JS, Zou X, Xia C, Sun K, Yang Z, Li H, et al: Changing cancer survival in China during 2003-15: A pooled analysis of 17 population-based cancer registries. Lancet Glob Health 6: e555-e567, 2018.

4. Dai W, Li Y, Mo S, Feng Y, Zhang L, Xu Y, Li Q and Cai G: A robust gene signature for the prediction of early relapse in stage I-III colon cancer. Mol Oncol 12: 463-475, 2018.

5. Chen K, Koe CT, Xing ZB, Tian X, Rossi F, Wang C, Tang Q, Zong W, Hong WJ, Taneja R, et al: Arl2- and Msps-dependent microtubule growth governs asymmetric division. J Cell Biol 212: 661-676, 2016.

6. Ozdemir ES, Jang H, Gursoy A, Keskin O and Nussinov R: Arl2-mediated allosteric release of farnesylated KRas4B from shuttling factor PDEd. J Phys Chem B 122: 7503-7513, 2018.

7. Stettin D, Waldmann A, Wolters M, Trunz B, Schauder P and Hahn A: Infection with Helicobacter pylori-outcome of a cross-sectional investigation. Dtsch Med Wochenschr 132: 2677-2682, 2007 (In German).

8. Zhang $\mathrm{F}$ and Cheong JK: The renewed battle against RAS-mutant cancers. Cell Mol Life Sci 73: 1845-1858, 2016.

9. Cox AD, Fesik SW, Kimmelman AC, Luo J and Der CJ: Drugging the undruggable RAS: Mission possible? Nat Rev Drug Discov 13: 828-851, 2014.

10. Stephen AG, Esposito D, Bagni RK and McCormick F: Dragging ras back in the ring. Cancer Cell 25: 272-281, 2014.

11. Wang Y, Guan G, Cheng W, Jiang Y, Shan F, Wu A, Cheng P and Guo Z: ARL2 overexpression inhibits glioma proliferation and tumorigenicity via down-regulating AXL. BMC Cancer 18: 599, 2018.

12. Li HJ, Sun XM, Li ZK, Yin QW, Pang H, Pan JJ, Li X and Chen W: LncRNA UCA1 promotes mitochondrial function of bladder cancer via the MiR-195/ARL2 signaling pathway. Cell Physiol Biochem 43: 2548-2561, 2017.

13. Béghin A, Matera E, Brunet-Manquat S and Dumontet C: Expression of Arl2 is associated with p53 localization and chemosensitivity in a breast cancer cell line. Cell Cycle 7: 3074-3082, 2008. 
14. Hass HG, Vogel U, Scheurlen M and Jobst J: Gene-expression analysis identifies specific patterns of dysregulated molecular pathways and genetic subgroups of human hepatocellular carcinoma. Anticancer Res 36: 5087-5096, 2016.

15. Peng R, Men J, Ma R, Wang Q, Wang Y, Sun Y and Ren J: miR-214 down-regulates ARL2 and suppresses growth and invasion of cervical cancer cells. Biochem Biophys Res Commun 484: 623-630, 2017.

16. Martinelli E, Martini G, Cardone C, Troiani T, Liguori G, Vitagliano D, Napolitano S, Morgillo F, Rinaldi B Melillo RM, et al: AXL is an oncotarget in human colorectal cancer. Oncotarget 6: 23281-23296, 2015.

17. Uribe DJ, Mandell EK, Watson A, Martinez JD, Leighton JA, Ghosh S and Rothlin CV: The receptor tyrosine kinase AXL promotes migration and invasion in colorectal cancer. PLoS One 12: e0179979, 2017.

18. Bläker H: Grading of tumors in the tubular digestive tract: Esophagus, stomach, colon and rectum. Pathologe 37: 293-298, 2016 (In German)

19. Livak KJ and Schmittgen TD: Analysis of relative gene expression data using real-time quantitative PCR and the 2(-Delta Delta C(T)) method. Methods 25: 402-408, 2001.

20. Hou P, Li L, Chen F, Chen Y, Liu H, Li J, Bai J and Zheng J: PTBP3-mediated regulation of ZEB1 mRNA stability promotes epithelial-mesenchymal transition in breast cancer. Cancer Res 78: 387-398, 2018.

21. Qiu $\mathrm{C}, \mathrm{Bu} \mathrm{X}$ and Jiang Z: Protocadherin-10 acts as a tumor suppressor gene, and is frequently downregulated by promoter methylation in pancreatic cancer cells. Oncol Rep 36: 383-389, 2016.

22. Tohidi F, Sadat SM, Bolhassani A and Yaghobi R: Construction and production of HIV-VLP harboring MPER-V3 for potential vaccine study. Curr HIV Res 15: 434-439, 2017.

23. Seetharaman S and Etienne-Manneville S: Microtubules at focal adhesions-a double-edged sword. J Cell Sci 132: jcs232843, 2019

24. van Vuuren RJ, Botes M, Jurgens T, Joubert AM and van den Bout I: Novel sulphamoylated 2-methoxy estradiol derivatives inhibit breast cancer migration by disrupting microtubule turnover and organization. Cancer Cell Int 19: 1, 2019.

25. Galmarini CM, Martin M, Bouchet BP, Guillen-Navarro MJ, Martínez-Diez M, Martinez-Leal JF, Akhmanova A and Aviles P: Plocabulin, a novel tubulin-binding agent, inhibits angiogenesis by modulation of microtubule dynamics in endothelial cells. BMC Cancer 18: 164, 2018.
26. Long LM, He BF, Huang GQ, Guo YH, Liu YS and Huo JR: microRNA-214 functions as a tumor suppressor in human colon cancer via the suppression of ADP-ribosylation factor-like protein 2. Oncol Lett 9: 645-650, 2015.

27. Zhou C, Cunningham L, Marcus AI, Li Y and Kahn RA: Arl2 and Arl3 regulate different microtubule-dependent processes. Mol Biol Cell 17: 2476-2487, 2006.

28. Newman LE, Schiavon CR, Zhou C and Kahn RA: The abundance of the ARL2 GTPase and its GAP, ELMOD2, at mitochondria are modulated by the fusogenic activity of mitofusins and stressors. PLoS One 12: e0175164, 2017.

29. Song X, Akasaka H, Wang H, Abbasgholizadeh R, Shin JH, Zang F, Chen J, Logsdon CD, Maitra A, Bean AJ and Wang H: Hematopoietic progenitor kinase 1 down-regulates the oncogenic receptor tyrosine kinase AXL in pancreatic cancer. J Biol Chem 295: 2348-2358, 2020.

30. Bae CA, Ham IH, Oh HJ, Lee D, Woo J, Son SY, Yoon JH, Lorens JB, Brekken RA, Kim TM, et al: Inhibiting the GAS6/AXL axis suppresses tumor progression by blocking the interaction between cancer-associated fibroblasts and cancer cells in gastric carcinoma. Gastric Cancer 23: 824-836, 2020.

31. Vajkoczy P, Knyazev P, Kunkel A, Capelle HH, Behrndt S, von Tengg-Kobligk H, Kiessling F, Eichelsbacher U, Essig M, Read TA, et al: Dominant-negative inhibition of the Axl receptor tyrosine kinase suppresses brain tumor cell growth and invasion and prolongs survival. Proc Natl Acad Sci USA 103: 5799-5804, 2006.

32. Cheng P, Phillips E, Kim SH, Taylor D, Hielscher T, Puccio L, Hjelmeland AB, Lichter P, Nakano I and Goidts V: Kinome-wide shRNA screen identifies the receptor tyrosine kinase AXL as a key regulator for mesenchymal glioblastoma stem-like cells. Stem Cell Reports 4: 899-913, 2015.

This work is licensed under a Creative Commons Attribution-NonCommercial-NoDerivatives 4.0 International (CC BY-NC-ND 4.0) License. 